\title{
The Cutaneous Side Effects of Selective BRAF Inhibitors and Anti-CTLA4 Agents: the Growing Role of the Dermatologist in the Management of Patients with Metastatic Melanoma
}

\author{
Lisa Pappas-Taffer • Misha Rosenbach • Emily Y. Chu
}

Published online: 5 April 2013

(C) Springer Science+Business Media New York 2013

\begin{abstract}
Two new drugs - vemurafenib, a selective BRAF inhibitor (BRAFi), and ipilimumab, an anti-CTLA4 monoclonal antibody - were approved by the FDA in 2011 and have demonstrated a survival benefit in the treatment of metastatic melanoma. Cutaneous side effects are common with both medication classes, occurring in up to $92 \%$ of patients treated with BRAFis, and up to $69 \%$ of patients treated with antiCTLA4 therapy. Cutaneous eruptions associated with BRAFis include benign and malignant squamous and melanocytic proliferations, photosensitivity, morbilliform eruptions, diffuse keratosis pilaris, Grover's disease, seborrheic dermatitis, panniculitis, hand-foot skin reaction, epidermal cysts, and hair changes. Dermatologic findings associated with anti-CTLA4 agents include morbilliform eruptions, hair depigmentation, vitiligo, and alopecia areata. Given the array of skin eruptions occurring in melanoma patients receiving these agents, the role of the dermatologist in monitoring and treating these patients is paramount.
\end{abstract}

Keywords Melanoma $\cdot$ BRAF inhibitors · Vemurafenib . Dabrafenib · Anti-CTLA4 · Ipilimumab · Squamous cell carcinoma $\cdot$ Keratoacanthoma $\cdot$ Verrucous keratoses . Photosensitivity · Keratosis pilaris · Grover's disease · Acantholytic dyskeratosis · Seborrheic dermatitis .

Lisa Pappas-Taffer wrote the manuscript. Misha Rosenbach and Emily Chu edited and provided photographs for the manuscript.

L. Pappas-Taffer $(\varangle) \cdot$ M. Rosenbach $\cdot$ E. Y. Chu

Department of Dermatology, Perelman School of Medicine,

The University of Pennsylvania, Philadelphia, PA 19104, USA

e-mail: lisakpappas@gmail.com
Panniculitis $\cdot$ Hand-foot skin reaction $\cdot$ Palmar-plantar hyperkeratosis $\cdot$ Cysts $\cdot$ Alopecia $\cdot$ Curly hair $\cdot$ Nevi $\cdot$ Basal cell carcinoma $\cdot$ Ipilimumab $\cdot$ Hair depigmentation $\cdot$ Vitiligo

\section{Introduction}

BRAFi-associated eruptions can be attributed to either the inhibition or paradoxical activation of the RAS/RAF/ MEK/ERK signaling cascade, which regulates cellular proliferation and differentiation [1]. In contrast, anti-CTLA4associated eruptions are largely attributed to the inhibition of regulatory immune mechanisms resulting in enhanced $\mathrm{T}$ cell response against self-antigens [2]. We review the literature describing these diverse cutaneous reactions, characterizing their clinical and histologic appearance, frequency of occurrence, timing of onset, and treatment options.

\section{Selective V600E-BRAF Inhibitors}

Introduction

BRAF is a serine/threonine kinase component of the RAS/RAF/MEK/ERK signaling cascade, also known as the mitogen-activating protein kinase (MAPK) pathway, involved in cellular proliferation and differentiation. At least $50 \%$ of all melanomas harbor an activating BRAF gene mutation, with a V600E substitution (valine $\rightarrow$ glutamic acid at position 600 ) occurring in the majority of cases [3, 4]. A V600E-BRAF mutation causes sustained activation of the MAPK pathway, resulting in cellular hyperproliferation [1]. 
The discovery of V600E-BRAF mutation in melanoma [3], and the ability to target this mutation therapeutically, has revolutionized the treatment of metastatic melanoma. In August 2011, V600E-BRAF targeting agent, vemurafenib, was approved by the Federal Drug Administration (FDA), and is becoming the standard of care for patients with V600E-BRAF-containing metastatic melanoma [5, 6]. Other selective BRAFis may be approved in the near future, including dabrafenib, which is completing phase III trials for use as a monotherapy, and as combination therapy with MEK inhibitor, trametinib [7, 8]. With the increasing use of BRAFis, and the introduction of combination therapies targeting different components of the MAPK pathway, it will be important for dermatologists to recognize the cutaneous side effects that occur in up to $92 \%$ of patients treated with BRAFis [9••]. Specific reactions are discussed in detail below.

\section{Squamous Proliferations}

\section{Cutaneous Squamous Cell Carcinoma (cSCC) and Keratoacantoma (KA)}

BRAFi-induced cSCCs present as KA-like exophytic nodules [10], inflamed verrucous papules [11••], or pink/red thin plaques [12••]; occurring most commonly

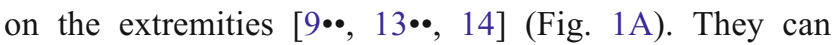
present with or without tenderness, and as single or multiple lesions $[9 \bullet \bullet, 11 \bullet \bullet, 12 \bullet \bullet]$. Risk factors for developing BRAFi-associated cSCC are unclear; however, a history of non-melanoma skin cancers [13••, 14] and an older age [11••] have been reported.

The mechanism underlying the formation of BRAFiassociated cSCCs is an area of active investigation. It is thought that BRAFis paradoxically activate MAPK signaling in cells that contain wild-type $B R A F$ by increased signaling through CRAF when BRAF is inhibited [15]. Recently, it has been shown that $R A S$ mutations are present at a higher rate in BRAFi-associated cSCCs compared with conventional cSCC (30-70\% compared with $3.2 \%$ ) [14, $16,17]$, with a mouse model demonstrating accelerated onset of cSCCs in the setting of cutaneous HRAS mutation and vemurafenib [14]. Thus, in the setting of BRAFi therapy, pre-existing $R A S$ mutations in keratinocytes (resulting from ultraviolet damage and/or viral infection) seem to play an important role in the development of cSCCs.

Histologically, BRAFi-associated cSCCs may be in situ or invasive, and often with a KA-type architecture [13••, 18•]. BRAFi-associated KAs differ histologically from conventional KAs in that they lack the classic KA finding of neutrophilic/eosinophilic microabscesses [13••] (Fig. 1B).

In clinical trials and subsequent dermatology-reported cohorts, cSCCs and KAs are reported to occur in 18-33\% of patients treated with vemurafenib, 6-20\% with dabrafenib, and $2-11 \%$ with dabrafenib/trametinib combination (Table 1 ), with the median time to onset being 8 weeks (2-36 weeks) [5, 19] and 9.5-16 weeks [9••, 20], respectively, for vemurafenib
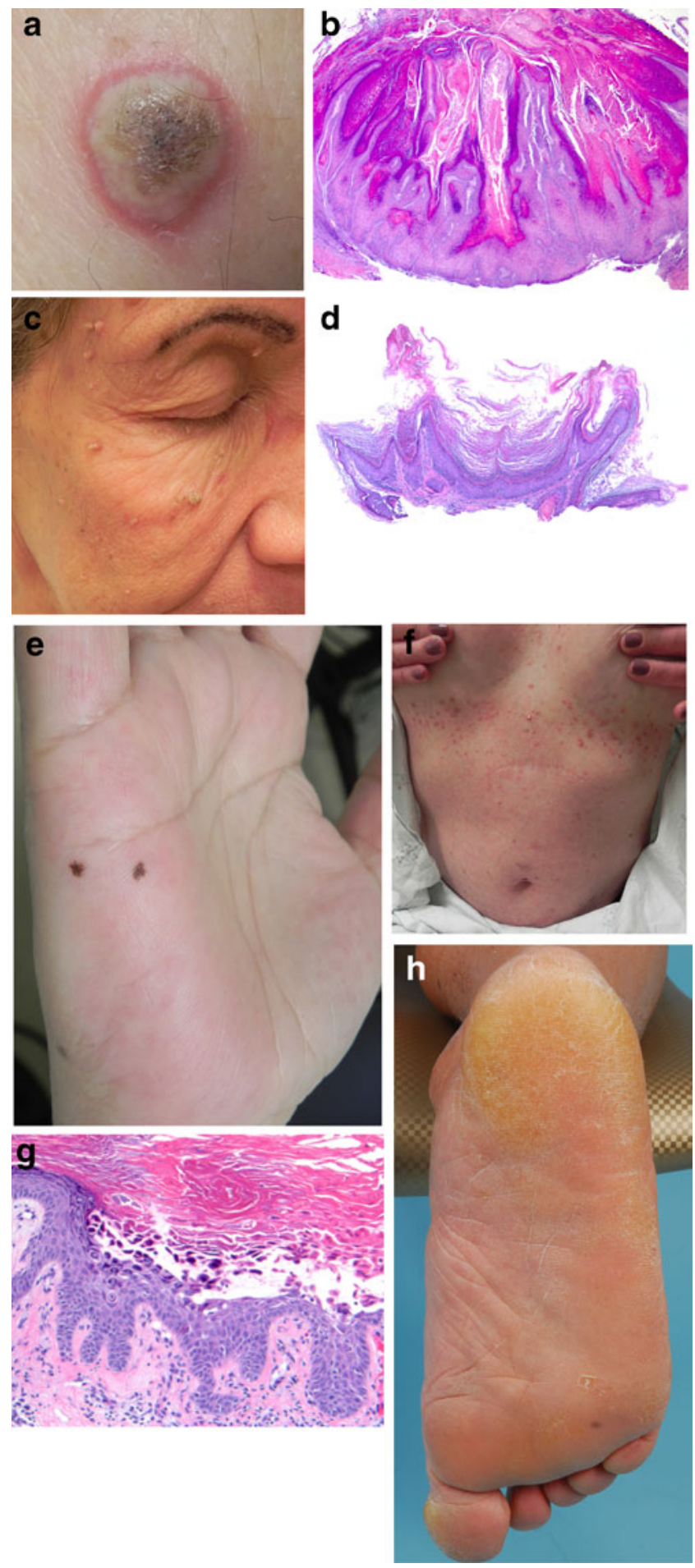

Fig. 1 (A) cutaneous squamous cell carcinoma (cSCCs); $(B)$ cSCC histopathology; $(C)$ verrucous keratoses (VKs); $(D)$ VK histopathology; $(E)$ new atypical nevi; $(F)$ Grover's disease; $(G)$ acantholytic dyskeratosis on histopathology; $(H)$ hand-foot skin reaction (HFSR) 
and dabrafenib, with even greater delay in onset with combination BRAF/MEKi therapy [21•].

To date, no studies have been performed to compare the efficacy and safety of different modalities for treating cSCC in the setting of BRAFi therapy. All clinical trials utilized excision of cSCCs. Subsequently, anecdotal treatment algorithms $[11 \bullet, 22 \bullet]$, and cases of successful treatment with non-surgical modalities have been reported (Table 2).

There is consensus among investigators that regular monitoring with routine full body skin exams is necessary $[9 \bullet \bullet$, $11 \bullet \cdot$, and promisingly, the addition of MEK inhibitors have been shown to reduce the frequency of cutaneous toxicities, including cSCCs/KAs [8].

\section{Verrucous Keratoses (VKs)}

Verrucous keratoses (VKs) (also referred to as warts, skin papillomas, and verrucous keratoses in the literature) are flesh-to-light pink colored verrucous papules that occur most commonly on the cheeks and nose [13••], and sometimes in an eruptive fashion $[11 \bullet \bullet]$. They may resemble warts, small KAs, or non-specific hyperkeratotic papules [9・•] (Fig. 1C).

Like cSCCs, VKs occur on both sun damaged and nonsun damaged skin [9••], and more commonly in older individuals (mean age 59) compared with younger individuals (median age 39) $(p=0.009)[9 \bullet \bullet]$. Histologically, VKs can resemble verruca vulgaris $[9 \cdot \bullet, 13 \cdot \bullet, 18 \bullet]$, but lack koilocytes or clumped keratohylin granules with negative HPV staining [13••, 23•]; hypertrophic actinic keratoses (hAKs), with $86 \%$ of VKs demonstrating atypia [9••]; or KAs [18•]; with VKs having an even higher frequency of RAS mutation than $\mathrm{KAs} / \mathrm{cSCCs}$ [23•]. Although most authors consider them to be benign entities, it has been suggested that they may instead represent a pre-malignant precursor to $\mathrm{cSCC}$ (Fig. 1D).

In clinical trials and subsequent dermatology-reported cohorts, VKs are reported to occur in $29-82 \%$ of patients treated with vemurafenib, 15-49\% of patients treated dabrafenib, and $4-17 \%$ of patients treated with combination dabrafenib/trametinib (Table 1), with a mean onset of 11 weeks (peaking at 6-12 weeks) [9・•]. Of note, in dabrafenib phase I-III clinical trials, benign keratoses were collectively reported as "hyperkeratosis" (which included acanthomas, acrochordons, actinic keratoses, seborrheic keratoses, and hyperkeratosis, lichenoid keratosis, keratosis pilaris, and palmoplantar dysesthesias), preventing accurate assessment of the frequency of these entities [7, 20, 24].

Evidence-based treatment recommendations and prognosis for BRAFi-induced VKs is scant. A few reports of institution-specific treatments have been reported (Table 2). In our experience, having a low threshold for diagnostic skin biopsy is prudent, given the clinical similarities of cSCCs and VKs.
Hypertrophic Actinic Keratoses (hAKs)

Hypertrophic Actinic Keratoses (hAKs) clinically overlap in appearance with VKs and verruca vulgaris. hAKs were not noted in vemurafenib clinical trials, and similar to VKs, were reported collectively with other cutaneous findings as "hyperkeratosis" in dabrafenib trials [7, 24]. In clinical trials and subsequent dermatology-reported cohorts, hAKs are reported to occur in $7-40 \%$ of patients treated with vemurafenib, $10 \%$ of those treated with dabrafenib, and $22 \%$ of patients treated with combination dabrafenib/trametinib (Table 1), with no reports regarding time to onset. Some authors note that hAKs may be overdiagnosed in the setting of BRAFi therapy [9・・]. Alternatively, Vks, Vks with atypia, hAKs, and KA/cSCC may represent a spectrum. Published recommendations are scarce for the treatment of hAKs in the setting of BRAFi therapy (Table 2).

\section{Seborrheic Keratoses (SKs)}

Seborrheic keratoses (SKs) are waxy, "stuck-on," benign squamous epidermal growths that range in color from dark brown to pink/white. SKs were reported to occur in all dabrafenib phase I-III studies, classified under "hyperkeratoses" [7, 24] (Table 1). Subsequently, Anforth et al. reported eruptive SKs occurring in $34 \%$ (14/41) of patients in the setting of their dabrafenib-treated cohort $[9 \bullet \bullet]$. There are no reports of treatment, nor are there reports of SK regression with treatment withdrawal (Table 2).

\section{Other Neoplastic Proliferations}

\section{Melanocytic Proliferations (Nevi and Melanomas)}

Over the last year, cases of preexisting melanocytic nevi developing atypical features, new eruptive nevi $[9 \cdot \bullet, 13 \cdot \bullet$, $25 \bullet, 26 \bullet \cdot 27 \bullet]$, and new primary melanomas $[18 \bullet, 25 \bullet$, $26 \bullet \cdot$, have been reported in the context of vemurafenib therapy (Fig. 1E). In 2011, Dalle et al. first reported the detection of new atypical melanocytic nevi (5/6 found to be early wildtype BRAF-containing melanomas) occurring 4-12 weeks after patients started vemurafenib therapy [25•]. Chapman et al. subsequently reported that $<1 \%(5 / 464)$ of patients treated in phase II and III vemurafenib trials developed new primary melanomas [25•]. In dabrafenib trials, $1.6 \%(3 / 187)$ of patients were reported to develop new primary melanomas [7]. In clinical trials and subsequent dermatology-reported cohorts, $1-32 \%$ of patients were reported to develop new nevi among dabrafenib-treated patients (Table 1).

Interestingly, new nevi and primary melanomas that arise in the setting of BRAFi-therapy consistently lack the V600E$B R A F$ mutation - in contrast to de novo acquired nevi in the general population where this mutation is found to occur in up to $80 \%$ of lesions $[25 \bullet, 26 \bullet$. Haenssle et al. characterized 
Table 1 Frequency and time to onset of cutaneous toxicities associated with selective BRAF inhibitors and anti-CLTA-4 agents

\begin{tabular}{|c|c|c|c|c|}
\hline & \multicolumn{4}{|l|}{ Selective BRAF Inhibitors } \\
\hline & \multicolumn{3}{|c|}{ ( $\%$ cases reported in literature) } & \multirow[b]{2}{*}{ Time to Onset } \\
\hline & Vemurafenib (V) & Dabrafenib (D) & BRAF/MEK & \\
\hline \multirow[t]{2}{*}{$c S C C / K A$} & $18-63 \%$ (124 cases) & $6-20 \%(62$ cases $)$ & $2-11 \%$ (7 cases) & $\begin{array}{l}V: \text { Mean } 8(2-32) \\
\quad \text { weeks }[13 \cdot \bullet, 19]\end{array}$ \\
\hline & & & & $\begin{array}{l}\text { D: Median } 9.5-16 \\
\text { weeks }[9 \cdot \bullet, 20]\end{array}$ \\
\hline \multirow[t]{4}{*}{ Trials (Phase I-III) } & III [5]: $18 \%(61 / 336)$ & III [8]: $19 \%(10 / 53)$ & $\begin{array}{l}\mathrm{I} / \mathrm{II}[8]: 2-7 \% \\
\quad(1-4 / 55)^{\wedge}\end{array}$ & \\
\hline & II [19]: $26 \%(34 / 132)$ & III [7]: 6 \% (12/187) & & \\
\hline & I [55]: $31 \%(10 / 32)$ & II [24]: $6 \%(11 / 172)$ & & \\
\hline & & I [20]: $11 \%(20 / 184)$ & & \\
\hline Chu et al. [13••] & $63 \%(7 / 11)$ & 1 case & $0 \%(0 / 2)$ & \\
\hline Huang et al. $[12 \bullet \bullet]$ & $33 \%(5 / 13)$ & & & \\
\hline Sinha et al. $[11 \bullet \bullet]$ & $30 \%(3 / 10)$ & & & \\
\hline Mattai et al. $[21 \bullet]$ & $26 \%(4 / 15)$ & & $11 \%(2 / 18)$ & \\
\hline Anforth et al. $[9 \cdot \bullet]$ & & $20 \%(8 / 41)$ & & \\
\hline Verrucous Keratoses & $29-82 \%(58$ cases $)$ & $15-49 \%(29$ cases $*)$ & $4-17 \%$ (11 cases) & $\begin{array}{l}\text { V:Mean } 11(6-12) \\
\text { weeks }[9 \cdot 0]\end{array}$ \\
\hline \multirow[t]{3}{*}{ Trials } & III [5]: NR & III [8]: $15 \%(8 / 53)$ & $\mathrm{I} / \mathrm{II}[8]: 4-7 \%(2-4 / 55)^{\wedge}$ & \\
\hline & II [19]: $29 \%(38 / 132)$ & II [24]III [7]: reported* & & \\
\hline & I [55]: NR & I [20]: NR & & \\
\hline Chu et al. $[13 \cdot \bullet]$ & $82 \%(9 / 11)$ & 1 case & 2 cases $(2 / 2)$ & \\
\hline Huang et al. $[12 \bullet \bullet]$ & 1 case report & & & \\
\hline Sinha et al. $[11 \bullet \bullet]$ & $30 \%(3 / 10)$ & & & \\
\hline Mattai et al. [21•] & $46.7 \%(7 / 15)$ & & $17 \%(3 / 18)$ & \\
\hline Anforth et al. $[9 \cdot \bullet]$ & & $49 \%(20 / 41)$ & & \\
\hline Actinic keratoses & $7-40 \%$ (7 cases $)$ & $10 \%(18$ cases *) & $22 \%$ (4 cases) & $N R$ \\
\hline \multirow[t]{3}{*}{ Trials } & I-III $[5,19,55]:$ NR & III [8]: NR & I/II [8]: NR & \\
\hline & & II [24]III [7]:reported* & & \\
\hline & & I [20]: $10 \%(18 / 184)$ & & \\
\hline Huang et al. $[12 \bullet \bullet]$ & $7 \%(1 / 15)$ & & & \\
\hline Mattai et al. [21•] & $40 \%(6 / 15)$ & & $22 \%(4 / 18)$ & \\
\hline Anforth et al. $[9 \cdot \bullet]$ & & $10 \%(4 / 41)$ & & \\
\hline Seborrheic Keratoses & $N R$ & $34 \%$ (reported $*)$ & $N R$ & $N R$ \\
\hline \multirow[t]{2}{*}{ Trials } & I-III $[5,19,55]:$ NR & III [8]: NR & $\mathrm{I} / \mathrm{II}[8]: \mathrm{NR}$ & \\
\hline & & I-III [7, 20, 24]: reported* & & \\
\hline Anforth et al. $[9 \bullet \bullet]$ & & $34 \%(14 / 41)$ & & \\
\hline $\begin{array}{l}\text { New/changing Melanocytic } \\
\text { lesions }\end{array}$ & $\begin{array}{l}<1 \% \text { new melanomas } \\
\text { (11cases); nevi (3 cases) }\end{array}$ & $\begin{array}{c}1.6 \% \text { melanomas (14 cases); } \\
1-32 \% \text { nevi }(22 \text { cases })\end{array}$ & $N R$ & 4-12 weeks $[25 \bullet]$ \\
\hline \multirow[t]{5}{*}{ Trials } & III [5]: NR & III [7]: $1.6 \%(3 / 187)$ & $\mathrm{I} / \mathrm{II}[8]: \mathrm{NR}$ & \\
\hline & II/III [25]: <1 \%(5/464) & New melanomas & & \\
\hline & & II [24]: $1 \%(1 / 172)$ & & \\
\hline & & New nevi & & \\
\hline & & I [20] III [8]: NR & & \\
\hline Anforth et al. $[9 \cdot \bullet]$ & & $32 \%(13 / 41)-$ nevi & & \\
\hline Zimmer et al. [26••] & & $\begin{array}{l}11 \text { melanoma, } 8 \text { dysplastic } \\
\text { nevi }\end{array}$ & & \\
\hline
\end{tabular}


Table 1 (continued)

\begin{tabular}{|c|c|c|c|c|}
\hline & \multicolumn{4}{|c|}{ Selective BRAF Inhibitors } \\
\hline & \multicolumn{3}{|c|}{ ( $\%$ cases reported in literature) } & \multirow[b]{2}{*}{ Time to Onset } \\
\hline & Vemurafenib (V) & Dabrafenib (D) & BRAF/MEK & \\
\hline Harvey et al $[18 \bullet]$ & $\begin{array}{l}1 \text { melanoma } \\
1 \text { nevus }\end{array}$ & & & \\
\hline Dalle et al. $[25 \bullet]$ & $\begin{array}{l}5 \text { melanoma } \\
1 \text { nevus }\end{array}$ & & & \\
\hline Haenssle et al. [27•] & New nevi & & & \\
\hline Chu et al. $[13 \bullet \bullet]$ & 1 case nevi & & & \\
\hline $\begin{array}{l}\text { Basal Cell } \\
\text { Carcinoma }\end{array}$ & $6-13 \%$ (11 cases $)$ & $2-5 \%$ (4 cases $)$ & $6 \%(1$ case $)$ & $N R$ \\
\hline \multirow[t]{3}{*}{ Trials } & III [5]: NR & III [8]: NR & I/II [8]: NR & \\
\hline & II [19]: $6 \%(8 / 132)$ & III [7]: $2 \%(4 / 187)$ & & \\
\hline & I [55]: NR & I-II $[20,24]:$ NR & & \\
\hline Mattei et al. $[21 \bullet]$ & $13 \%(2 / 15)$ & & $6 \%(1 / 18)$ & \\
\hline Anforth et al. $[9 \bullet \bullet]$ & & $5 \%(2 / 41)$ & & \\
\hline Harvey et al [18•] & 1 case & & & \\
\hline Photosensitivity & $12-80 \%$ (201 cases) & $0-3 \%$ (5 cases $)$ & $17 \%$ (3 cases) & $\begin{array}{l}15 \min -24 \text { hours } \\
{[11 \bullet \bullet]}\end{array}$ \\
\hline \multirow[t]{4}{*}{ Trials } & III [5]: $12 \%(40 / 336)$ & III [8]: NR & I/II [8]: NR & \\
\hline & II [19]: $52 \%(69 / 132)$ & III [7]: $3 \%(5 / 187)$ & & \\
\hline & I [55]: $16 \%(5 / 32)$ & II [24]: NR & & \\
\hline & & I [20]: $0 \%(0 / 184)$ & & \\
\hline Sinha et al. $[11 \bullet \bullet]$ & $80 \%(8 / 10)$ & & & \\
\hline Mattai et al. [21•] & $67 \%(10 / 15)$ & & $17 \%(3 / 18)$ & \\
\hline Anforth et al. $[9 \bullet \bullet]$ & & $0 \%(0 / 43)$ & & \\
\hline Zimmer et al. [43] & 1 case (pic) & & & \\
\hline Rash & $18-70 \%$ (147 cases $)$ & $4-36 \%$ ( 25 cases $)$ & $20-27 \%$ (26 cases) & $\begin{array}{c}\text { Mean 1-2 weeks } \\
{[11 \bullet \bullet]}\end{array}$ \\
\hline \multirow[t]{4}{*}{ Trials } & III [5]: $18 \%(61 / 336)$ & III [8]: 36 \% (19/53) & $\begin{array}{c}\text { I/II [8]: } 20-27 \% \\
(11-15-55)^{\wedge}\end{array}$ & \\
\hline & II [19]: $52 \%(69 / 132)$ & III [7]: NR & & \\
\hline & I [55]: $25 \%(8 / 32)$ & II [24]: 4 \% (6/172) & & \\
\hline & & I [20]: NR & & \\
\hline Sinha et al. $[11 \bullet \cdot]$ & $70 \%(7 / 10)$ & & & \\
\hline Infante et al. [37•] & & & $20 \%$ & \\
\hline Harvey et al $[18 \bullet]$ & 2 cases lichenoid & & & \\
\hline Hypersensitivity/morbilliform & $13.3 \%$ (11 cases) & $N R$ & & $N R$ \\
\hline Trials & I-III $[5,19,55]:$ NR & I-III $[7,8,20,24]:$ NR & I/II [8]: NR & \\
\hline Harding et al. $[31 \bullet \bullet]$ & $13.3 \%(9 / 13)$ & & & \\
\hline Novoa et al. [32•] & $2 / 2$ patients & & & \\
\hline ESS & $N R$ & $N R$ & $N R$ & $V: 1$ week $[34 \bullet]$ \\
\hline Trials & I-III $[5,19,55]:$ NR & I-III $[7,8,20,24]:$ NR & I/II [8]: NR & \\
\hline Story et al. [34•] & 1 case (face) & & & \\
\hline Novoa et al. [32•] & 1 case (leg) & & & \\
\hline
\end{tabular}


Table 1 (continued)

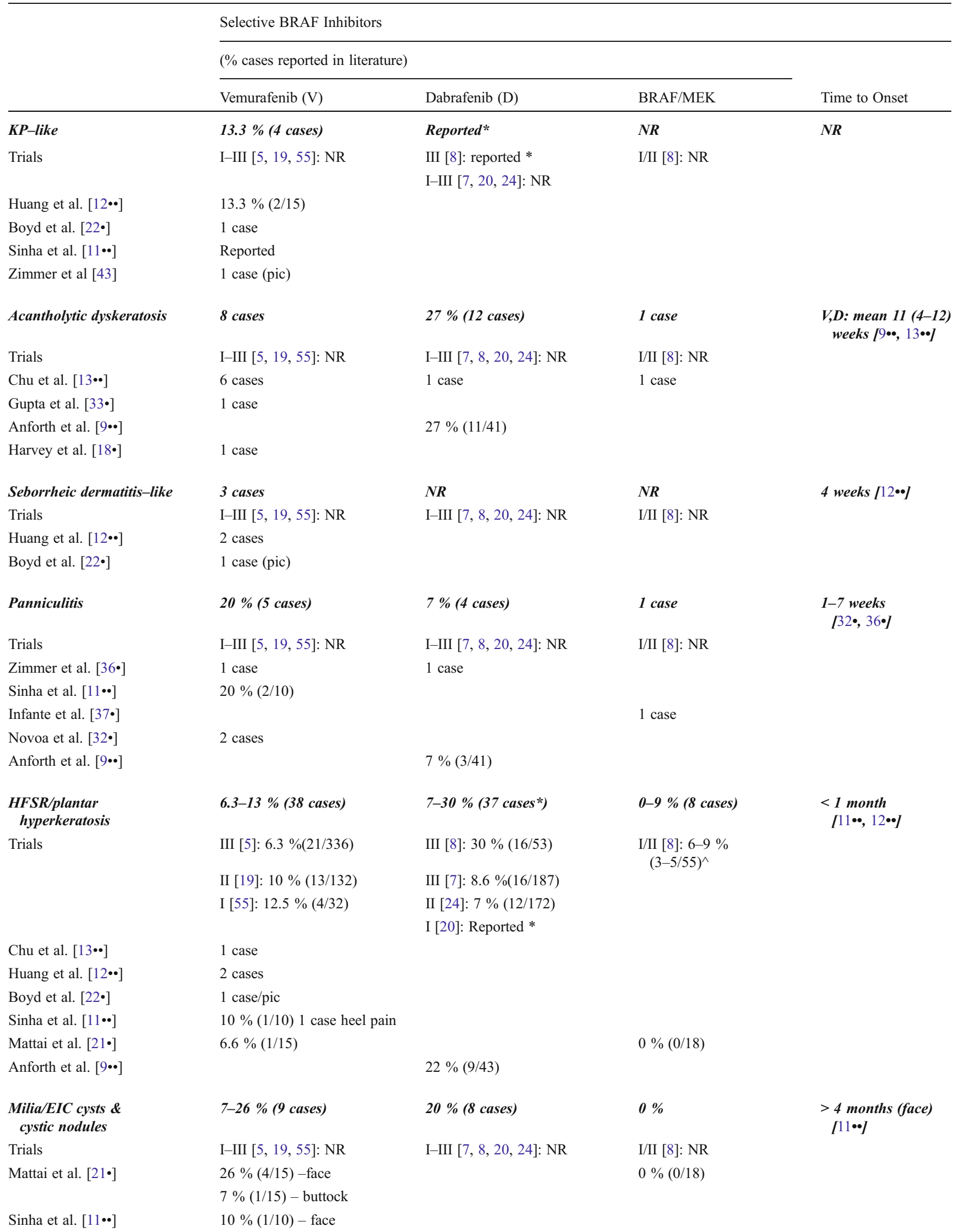


Table 1 (continued)

\begin{tabular}{|c|c|c|c|c|}
\hline & \multicolumn{4}{|c|}{ Selective BRAF Inhibitors } \\
\hline & \multicolumn{3}{|c|}{ ( $\%$ cases reported in literature) } & \multirow[b]{2}{*}{ Time to Onset } \\
\hline & Vemurafenib (V) & Dabrafenib (D) & BRAF/MEK & \\
\hline Anforth et al. $[9 \bullet \bullet]$ & & $20 \%(8 / 41)$ & & \\
\hline $\begin{array}{l}\text { Acneiform or } \\
\text { papulopulstular }\end{array}$ & $6.7 \%(1$ case $)$ & $4-7 \%$ (5 cases) & $11-17 \%(15$ cases $)$ & $N R$ \\
\hline \multirow[t]{2}{*}{ Trials } & I-III $[5,19,55]:$ NR & III [8]: 4 \% (2/53) & $\begin{array}{l}\text { I/II [8]: } 11-16 \% \\
(6-9 / 55)^{\wedge}\end{array}$ & \\
\hline & & I-III $[7,20,24]:$ NR & & \\
\hline Anforth et al. $[9 \bullet \bullet]$ & & $7 \%(3 / 41)$ & & \\
\hline Mattai et al. [21•] & $6.7 \%(1 / 15)$ & & $17 \%(3 / 18)$ & \\
\hline Huang et al. [12••] & 1 case & & & \\
\hline Boyd et al. [22•] & 2 cases (pics) & & & \\
\hline Alopecia & $36-70 \%$ (55 cases) & $1-34 \%$ (28 cases) & $5-9 \%$ (8 cases $)$ & $\begin{array}{l}\text { Mean } 134 \text { days } \\
{[11 \cdot 0]}\end{array}$ \\
\hline \multirow[t]{3}{*}{ Trials } & III [5]: NR & III [8]: 34 \% (18/53) & I/II [8]: 5-9 \% $(3-5 / 55)^{\wedge}$ & \\
\hline & II [19]: $36 \%(48 / 132)$ & II [24]: $1 \%(2 / 172)$ & & \\
\hline & I [55]: NR & I [20]III [7]: NR & & \\
\hline Sinha et al. $[11 \bullet \bullet]$ & $70 \%(7 / 10)$ & & & \\
\hline Anforth et al. $[9 \bullet \bullet]$ & & $20 \%(8 / 41)$ & & \\
\hline $\begin{array}{l}\text { Follicular erythema } \\
+ \text { hyperkeratosis }\end{array}$ & & $9 \%$ (4 cases) & & \\
\hline Anforth et al. $[9 \bullet \bullet]$ & & $9 \%(4 / 41)$ & & \\
\hline Curling of hair & & $17 \%$ (7 cases) & & \\
\hline Anforth et al. $[9 \bullet \bullet]$ & & $17 \%(7 / 41)$ & & \\
\hline
\end{tabular}

\section{ANTI-CTLA4 AGENTS}

$\%$ (\# cases reported in literature)

\begin{tabular}{|c|c|c|c|c|}
\hline & Ipilimumab Monotherapy & Ipilimumab + Cancer Vaccine & Ipilimumab + Decarbazine & Time to Onset \\
\hline Skin Toxicity & $\begin{array}{l}35-69 \%(2-3 \% \text { severe }) \\
{[39,46]}\end{array}$ & $40 \%$ & $49 \%$ & $\begin{array}{c}>3.6 \text { weeks } \\
\text { [44-46] }\end{array}$ \\
\hline Trials & $\begin{array}{l}\text { III [39]: } 43 \%(57 / 131) \\
\text { II [56]: } 69 \%(35 / 51) \\
\text { II [45]: } 35 \%(74 / 214) \\
\text { II [46]: } 49 \%(79 / 155) \\
\text { II [44]: } 68 \%(39 / 57)\end{array}$ & III [39]: $40 \%(152 / 380)$ & $\begin{array}{l}\text { III [52]: } 49 \% \\
(121 / 247)\end{array}$ & \\
\hline Rash & $\begin{array}{l}14-68 \% \text {, life-threatening } \\
3 \% \text { (128 cases })\end{array}$ & $4-100 \%$ (109 cases) & $\begin{array}{l}22.3 \%(1 \% \text { severe }) \\
\text { [52] (55 cases) }\end{array}$ & $\begin{array}{l}\text { Median 3-4 (0-17) } \\
\text { weeks [40, 42••, } \\
50 \bullet, 51]\end{array}$ \\
\hline Trials & $\begin{array}{l}\text { III [39]: } 19 \%(25 / 131) \\
\text { II [56]: Immune: } 33 \%(17 / 51) \text {; } \\
\text { Gen rash: } 37 \%(19 / 31) \\
\text { II [45]: } 17 \%(36 / 214) \\
\text { II [46]: NR } \\
\text { II [44]: } 68 \%(39 / 57)\end{array}$ & III [39]: $18 \%(67 / 380)$ & $\begin{array}{l}\text { III [52]: } 22 \% \\
\quad(55 / 247)\end{array}$ & \\
\hline Jaber et al. $[42 \bullet \bullet]$ & $14 \%(9 / 63)$ & & & \\
\hline
\end{tabular}


Table 1 (continued)

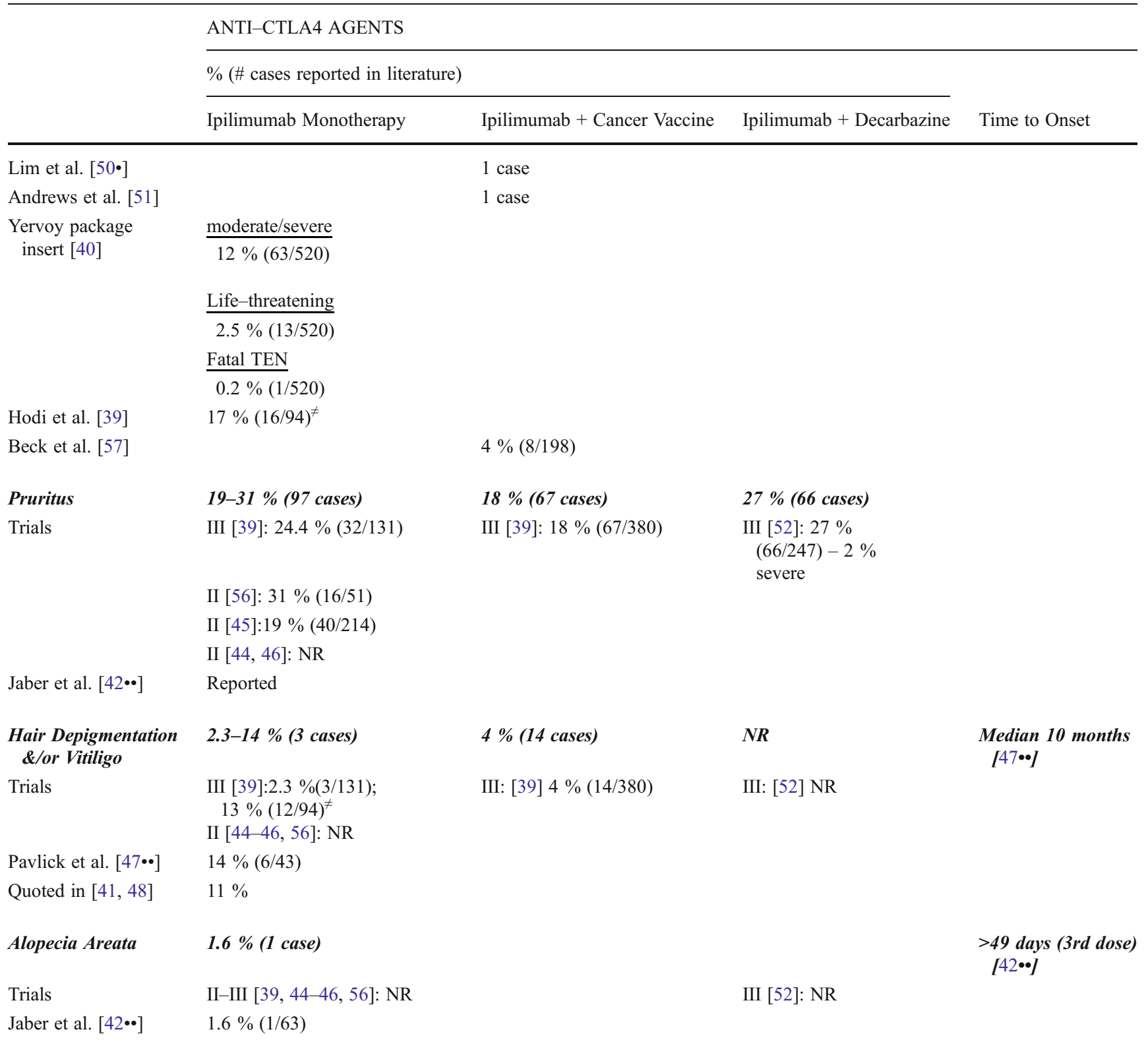

*Hyperkeratosis was a collective category of cutaneous eruptions in dabrafenib phase I-III trials. In phase I trial, it included: wart, seborrheic keratosis, palmoplantar erythrodysesthia (48/184 patients). In phase II trial, it included: actinic keratoses, seborrheic keratoses, and skin papillomas (15/172 patients). In phase III trial, it included: phase II trial + lichenoid keratoses and keratosis pilaris $(25 / 187)$

${ }^{\wedge}$ Based on $1 \mathrm{mg}$ vs. $2 \mathrm{mg}$ MEK inhibitor (trametinib) dose

${ }^{\neq}$Reported as residual effects in patients surviving 2 years: injection site reactions, vitiligo, rash and pruritus

Italics summary frequencies, Underline differentiate severity of eruptions

three major pattern changes on dermoscopy in dynamically changing nevi, which were hypothesized to correlate with $B R A F$ mutational status. These pattern changes included: 1) involuting nevi; 2) darkening/enlarging nevi; and 3) multiple nevi. The latter two patterns were found to contain WT-BRAF, with the first pattern of involution hypothesized to occur due to those nevi harboring the V600E-BRAF mutation [27•].
Although not explicitly stated in the literature, it is assumed that re-excision of melanoma and severely dysplastic nevi occurred in clinical trials (Table 2). There is consensus that all patients should have careful skin evaluations prior to and during BRAFi therapy by a dermatologist $[27 \bullet, 28,29]$, with frequent surveillance of patients with preexisting atypical nevi or very sun damaged skin [29]. It has been suggested that skin monitoring 
Table 2 Treatment algorithm for cutaneous toxicity to new melanoma drugs; based on reported successful modalities in the literature

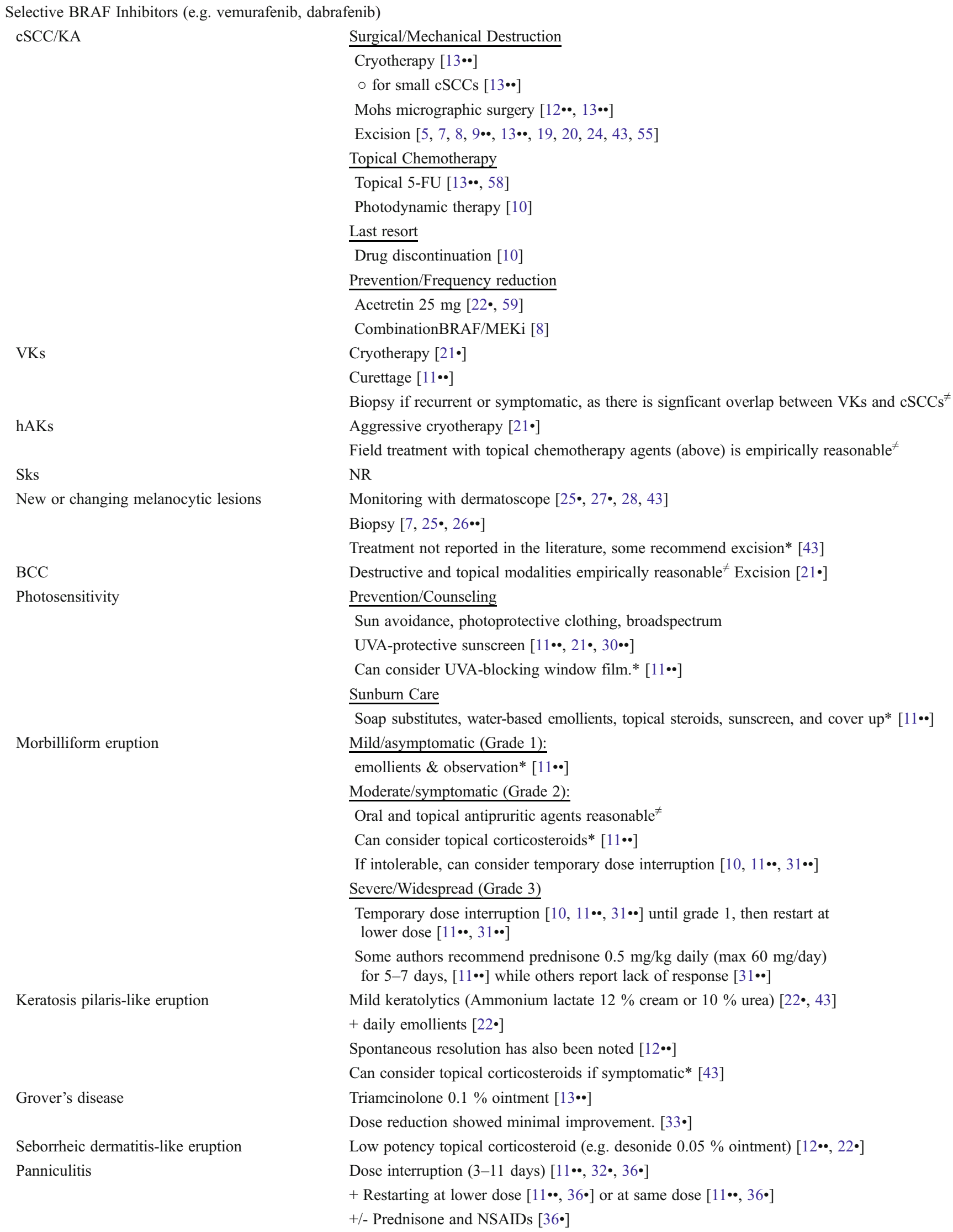


Table 2 (continued)

HFSR/hyperkeratosis

Milia/cysts
Self-resolution without treatment also reported [9••]

Empirically reasonable to treat symptomatically according to algorithms for HFSR treatment in the setting of multikinase inhibitors (e.g. sorafenib) $[22 \bullet, 35]^{\neq *}$

- Preventative strategies (skin care regimen, lifestyle modifcations to reduce friction and hot water)

- To target hyperkeratosis: can consider topical retinoids and/or keratolytic agents

- To target inflammation: can consider high-potency topical corticosteroids

- Pain control: $2 \%$ lidocaine, NSAIDs, GABA agonists (e.g. pregabalin), narcotics

Only self-resolution reported in literature $[11 \bullet, 12 \bullet \bullet, 22 \cdot]$

No treatment $[21 \bullet, 43]$

It is unclear if acne regimens are helpful, but have been proposed* [11••]

Topical retinoid $[22 \cdot]$

Acitretin $10 \mathrm{mg}$ daily [22•]

\begin{abstract}
Anti-CTLA4 Agents (e.g. ipilimumab)—Empirical

\section{RASH}

Monitoring

Monitor patients for signs and symptoms of dermatitis, such as rash and pruritus [40]

Consider any signs/symptoms of dermatitis to be immune-mediated [40]

Prophylactic application of topical corticosteroid not recommended [49]

Mild/Moderate(Grade 1-2)

No dose alteration [41, 43, 48, 49]

Symptomatic treatment

Oral antipruritics (diphyenhydramine or hydroxyzine) [41] or topical antipruritics

(e.g. polidocanol) [48]

AND

Use of topical steroid (e.g. betamethasone $0.1 \%$ cream) [41, 43, 48]

Or

Prednisone [51] $1 \mathrm{mg} / \mathrm{kg} /$ day dosing recommended by some [43]

Or

Later, urea-containing creams $[41,48]$

If persistent grade 2, can consider brief dose interruption [41]

Grade 3 (moderate/severe)

Dose interruption until grade 1 or less $[41,51]$

AND

4-week prednisone taper at $1 \mathrm{mg} / \mathrm{kg} /$ day [41]

Grade 4 (life-threatening)

AND

Systemic corticosteroids (1-2 mg/kg/day of prednisone equivalent) [40, 41, 43, 51]

Continue until controlled, then taper $>30$ days $[40,41,51]$

- May require weeks to months of high dose steroids or TNF-alpha blockers could be considered (used in the setting of non-skin immune-mediated toxicity) [60]

\footnotetext{
*Empirical recommendations in the literature

${ }^{\neq}$Author's input

underlined $=$ classes of treatment
}

should be enhanced with the use of a dermatoscope [25•, 27•, 28 ], with screening intervals of at least 2-3 months [6, 27•].

\section{Basal Cell Carcinoma (BCC)}

In the setting of BRAFi therapy, basal cell carcinomas (BCCs) have been reported to occur in 6-13\% of patients treated with vemurafenib, 2-5\% with dabrafenib, and $6 \%$ with dabrafenib/trametinib combination (Table 1). Time to onset was not reported. In one cohort, all BCCs were treated with excision [21•] (Table 2).

\section{Photosensitivity}

BRAFi-induced photosensitivity is characterized by a sunburn-like reaction, with or without blistering, upon 
minimal sun exposure. The onset to occurrence is reportedly within 15 minutes to 24 hours [11••], most commonly during outdoor exposure during summer months, but also indoors through window glass $[11 \bullet \cdot$. As UVA, but not UVB, light penetrates window glass, Dummer et al. photo tested five patients on vemurafenib therapy to further characterize BRAFi-induced photosensitivity. Their findings of a normal minimal erythema dose (MED) to UVB (i.e. the lowest dose of UV light needed to result in visible minimal erythema), but a strikingly reduced MED to UVA, was consistent with UVA-dependent phototoxicity [30••]. Furthermore, investigators found complete normalization of MED (UVA) on photo testing after applying a broadspectrum, UVA-tailored sunscreen, concluding that strict adherence to a UVA-protecting regimen should be able to prevent vemurafenib-induced photosensitivity $[30 \bullet \bullet$.

In clinical trials and subsequent dermatology-reported cohorts, photosensitivity is reported to occur in $12-80 \%$ of patients treated with vemurafenib, $0-3 \%$ with dabrafenib, and $17 \%$ with dabrafenib/trametinib combination (Table 1).

There is consensus among authors that counseling sun avoidance and photoprotection with a broad-spectrum UVAprotective sunscreen is important $[11 \bullet \bullet, 21 \bullet, 30 \bullet]$ (Table 2).

\section{Rash}

In clinical trials and subsequent dermatology-reported cohorts, "rash" was reported to occur in 18-70\% of patients treated with vemurafenib, 4-36\% with dabrafenib, and 20$27 \%$ with dabrafenib/trametinib combination (Table 1), often with a mean onset to appearance of 1-2 weeks after starting therapy $[11 \bullet \bullet$. A proportion of patients have concomitant pruritus (29\%), and not uncommonly develop fevers and arthralgias $(43 \%)$ [11••].

It is likely that reports of "rash" in clinical trials were over simplified, as subsequent reports of eruptions-including hypersensitivity/morbilliform eruptions, keratosis pilaris-like eruptions, acantholytic dyskeratotic eruptions (Grover's disease), panniculitis, and hand-foot skin reaction/palmar-plantar hyperkeratosis - have all been reported in the literature. Many of these clinically reported eruptions might represent spectrums of the same entity with similar histopathologic findings. For example, Sinha et al. subcategorized BRAFi-induced rashes as hypersensitivity/morbilliform eruptions versus exaggerated keratosis pilaris (KP)-like eruptions, with biopsy results demonstrating mild perifollicular and perivascular lymphocytic inflammation, with few eosinophilis in both $[11 \bullet \cdot$. However, these eruptions have only recently begun to be classified histologically, and two cases of BRAFi-associated lichenoid drug eruption have been noted [18•]. Further evaluation and classification clinically and histologically is needed.

In the literature, dose interruption for $7-11$ days $[10,11 \bullet$, $31 \bullet$ ], followed by restarting BRAFi at a lower dose, has been shown to reduce rash severity $[11 \cdot \bullet]$ and result in resolution $[10,31 \bullet \cdot$. There was no worsening of the rash with re-initiation of BRAFi at a lower dose, suggesting an underlying mechanism other than hypersensitivity [11••]. Although reports are limited, the use of systemic and topical corticosteroids appears to have variable utility $[11 \bullet, 31 \bullet \bullet]$ (Table 2).

\section{Morbilliform Eruption}

Sinha et al. described this eruption as "toxic erythema-like," clinically appearing identical to a classic drug-induced morbilliform eruption $[11 \bullet \cdot$. There is conflicting opinion as to whether this eruption is due to a hypersensitivity reaction or rather to up-regulation of alternative signaling pathways. Sinha et al. reports the latter, as patients were able to restart drug at a reduced dose, with a lack of severe reactions such as systemic drug hypersensitivity (e.g. DRESS syndrome) or Stevens-Johnson syndrome (SJS)/Toxic epidermal necrolysis $($ TEN) $[11 \bullet \cdot$. However, one case of SJS and one case of TEN, and cases of anaphylaxis in which drug continuation was not possible, were reported in the vemurafenib package insert [6]. In contrast, Harding et al. found a higher incidence of vemurafenib-induced morbilliform eruption $(9 / 13,69 \%)$, with hypersensitivity pattern on skin biopsy, in patients previously treated with ipilimumab $[31 \bullet \bullet]$. A greater severity and earlier onset was noted when vemurafenib was received within 4 weeks of ipilimumab use [31••]. Similarly, in a report of BRAFi-induced panniculitis, the presence of concomitant morbilliform eruption correlated with previous ipilimumab therapy [32•]. It has been hypothesized that a reduction of $\mathrm{T}$ cell inhibition by ipilimumab may predispose patients to hypersensitivity skin reactions with vemurafenib $[31 \bullet \cdot$. However, similar to the report from Sinha et al., eruptions resolved within 11 days of treatment interruption, with treatment re-initiation at lower doses without recurrence, and no reported impact in those treated with systemic corticosteroids [31••] (Table 2).

\section{Keratosis Pilaris (KP)-Like Eruption}

Keratosis pilaris (KP) is a common, benign cutaneous eruption characterized by erythematous-to-flesh colored keratotic, folliculocentric papules, most commonly on the extensor limbs. The dabrafenib phase III open label trial was the only clinical trial to note acquired $\mathrm{KP}$ in association with therapy, although the frequency was not reported [7]. Over the last year, there have been three dermatologic reports of diffuse or generalized KP-like eruptions in the context of vemurafenib or dabrafenib therapy $[11 \bullet \bullet, 12 \bullet, 22 \bullet]$, often occurring in the context of other cutaneous side effects $[12 \bullet \cdot]$. Although the true incidence is unclear, Huang et al. reported a generalized KP-like eruption in $13.3 \%$ ( 2 of 15 ) 
of patients treated with vemurafenib at their institution [12••]. In addition, Boyd et al. presented a patient with "diffuse spiny spicules," concluding this eruption was distinct from diffuse KP eruptions [22•]. Histology of these eruptions has not been reported to date.

Although treatment reports are scant, Boyd et al. reported improvement with ammonium lactate $12 \%$ cream and daily use of emollients [22•], while Huang et al. reported resolution after 3-4 weeks without dose adjustment [12••] (Table 2).

\section{Truncal Folliculocentric Eruptions with Acantholytic Dyskeratosis on Histology}

This eruption-manifesting as erythematous, scaly, 2-3 mm scattered papules, with or without crusting (Fig. 1F), characterized histologically as an acantholytic process with dyskeratosis (Fig. 1G) - has been reported with increasing frequency in the context of BRAFi therapy over the last year. Clinically and histologically, the eruption has been likened to Grover's disease (i.e. transient acantholytic dermatosis), an acquired pruritic, follicular eruption that occurs most commonly across the chest in middle-aged men. Similar to true Grover's disease, BRAFi-associated eruptions are asymptomatic to slightly pruritic, occurring more commonly in males than females (10:1), with a median age of 54 years (range, 30 83) [9••], demonstrating acantholysis with dyskeratosis on histology $[13 \bullet \bullet, 33 \bullet]$. The eruption most commonly affects the trunk, but a more diffuse distribution involving the extremities occurs in $20-27 \%$ of patients $[9 \bullet \cdot, 13 \bullet \cdot$. Disorders with acantholytic dyskeratosis are at increased risk for herpes simplex virus (HSV) superinfection, known as Kaposi varicelliform eruption. Notably, one case of preexisting Grover's disease worsened after starting vemurafenib, and was subsequently complicated by Kaposi varicelliform [33•]. A Grover's disease-like eruption was not specifically described in BRAFi clinical trials. However, multiple clinicopathologic reports of a Grover's disease-like eruption have been presented in the literature over the last year $[9 \bullet, 13 \bullet \bullet$, 18•, 33•]. To date, 21 cases of eruptions with acantholytic dyskeratosis have been reported - eight in the setting of

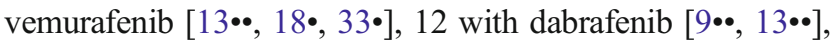
and one with combination BRAF/MEK inhibition [13••]. Although the frequency of this Grover's disease-like eruption is unclear, it was noted to occur in $27 \%$ (11/41) of patients treated with dabrafenib in one cohort $[9 \bullet \cdot$, with a mean time to presentation of 11 weeks [9••], ranging from 4-12 weeks $[13 \bullet \bullet]$. We have noted a good response to triamcinolone $0.1 \%$ ointment (Table 2).

\section{Seborrheic Dermatitis-Like Eruption}

Three cases of an erythematous facial eruption resembling seborrheic dermatitis have been reported in association with
BRAFi therapy $[12 \bullet, 22 \bullet]$, often in the setting of other cutaneous side effects $[12 \bullet$ ]. Huang et al. described one such eruption that occurred after 4 weeks of vemurafenib therapy, presenting as pruritic, erythematous patches across the forehead and chin, in a seborrheic distribution [12••]. Boyd et al. also described this eruption, but with more diffuse distribution, greater severity, and thicker yellow crust on the face, scalp and chest [22•]. Treatment with low-potency topical steroids has been used [12••, 22•], with improved pruritus noted over 3 weeks $[12 \bullet \bullet$ (Table 2).

\section{Eccrine Squamous Syringometaplasia (ESS)}

Eccrine squamous syringometaplasia (ESS) is a histologic diagnosis that is associated with eruptions most commonly caused by traditional chemotherapy agents. Two cases of ESS have been reported in association with BRAFi therapy in the literature $[32 \bullet, 34 \bullet]$. The first case was described as a pruritic facial eruption consisting of multiple $3-5 \mathrm{~mm}$ discrete, erythematous papules that began 1 week after starting vemurafenib therapy. A skin biopsy demonstrated ESS [34•]. The second case of ESS presented as a tender leg nodule, and a skin biopsy demonstrated ESS with a small vessel vasculitis (leukocytoclastic vasculitis) in the absence of ulceration or other reactive change [32•]. Most ESS spontaneously resolves after several months, and in the first case, significant reduction in the thickness and number of papules on the patient's face were noted 4 weeks after dose reduction $[34 \bullet]$.

\section{Follicular Papulopustular Eruption}

A papulopustular eruption has been reported by a single cohort group, in which $6.7 \%$ of patients treated with vemurafenib, and $16.7 \%$ of patients treated with combination dabrafenib/trametinib, developed these skin changes [21•]. This eruption is similar to EGFR-induced papulopustular eruption. A shared origin is hypothesized as a higher incidence is noted with the addition of a MEK inhibitor or with monotherapy MEK inhibitor [21•]. The treatment for this reaction mirrors the treatment algorithms recommended for EGFR inhibitors, including doxycycline, emollients, and topical corticosteroids [35].

Panniculitis

Over the last year, the development of panniculitis in the context of BRAFi therapy has been reported $[9 \bullet \bullet, 11 \bullet \bullet, 32 \bullet$, $36 \bullet, 37 \cdot]$, despite an absence of such reports in clinical trials. To date, ten cases of BRAFi-induced panniculitis have been reported in the literature, including five cases on vemurafenib, four cases on dabrafenib, and one case while on combination dabrafenib/trametinib $[9 \bullet \bullet, 11 \bullet \bullet, 32 \bullet, 36 \bullet$, 
37•]. The frequency of panniculitis has been reported to be $20 \%(2 / 10)$, and $7 \%(3 / 41)$ of patients treated with

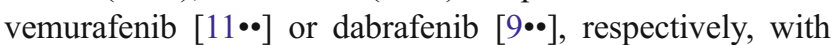
the time to onset typically within 4 weeks (range, 7 days to 7 weeks) [32•, 36•].

Clinically, patients develop painful, erythematous subcutaneous nodules, often starting on the shins, resembling erythema nodosum $[11 \bullet \bullet$. Lesions may remain limited to the lower extremities $[11 \bullet \bullet, 32 \bullet, 37 \bullet]$, or have a more widespread distribution involving upper and lower extremities $[32 \bullet, 36 \bullet$, and occasionally, shoulders or gluteal area [32•, 36•]. Although demographic information in the literature is limited, over $50 \%$ of patients reported were female, age 44 59 , with one male, age 26 , affected $[11 \bullet \bullet, 32 \bullet, 36 \bullet$. Histologically, of the ten cases of panniculitis reported, 8/10 were biopsy-proven, showing a septo-lobular panniculitis $[11 \bullet \bullet, 32 \bullet, 36 \cdot$, predominantly neutrophilic $[32 \bullet, 36 \bullet, 37 \bullet]$, with some reporting concomitant small vessel vasculitis [32•, 36•, 37•].

Reported associations have been mixed. Initial reports described panniculitis occurring in association with arthralgias (three cases) $[11 \bullet \bullet, 36 \bullet$. Since this time, cases have been reported to occur in the absence of arthralgias (four cases) $[9 \bullet \bullet, 11 \bullet \cdot$, with one report of fevers and chills in the context of combination BRAF/MEK inhibitor therapy [37•].

Rare reports of panniculitis-like presentation of melanoma metastases exist. Hence, in the setting of BRAFiinduced panniculitis, skin biopsy with melan-A staining should be considered [36•]. Dose interruption (ranging from 3 days to 11 days) $[11 \bullet \bullet, 32 \bullet, 36 \bullet$, followed by re-initiation at a lower dose $[11 \bullet \bullet, 36 \bullet$ or at the same dose $[11 \bullet \bullet, 36 \bullet$, has been shown to be effective in most cases [32•]. Zimmer et al. reported gradual resolution of lesions with dose interruption and symptomatic treatment (prednisone and/or NSAIDs) [36 ]. However, Anforth et al. reported three cases of self-resolving panniculitis $[9 \bullet \bullet]$, suggesting that this may be a self-limited process.

Although Sinha et al. reported both patients in their cohort being treated successfully with drug interruption then dose reduction, the authors suggest a treatment protocol for BRAFi-induced panniculitis that includes referral to dermatology for advice on the diagnosis and treatment, skin biopsy and blood tests; analgesics (paracetamol, NSAIDs), emollients and topical steroids; consideration for oral steroids; and consideration for interrupting BRAFi [11] (Table 2).

\section{Hand-Foot Skin Reaction (Palmar-Plantar Hyperkeratosis)}

BRAFi therapy has been reported to induce a palmar/plantar eruption; however, terminology in the literature varies. Terms

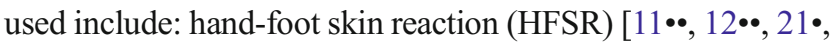
22•], palmar-plantar hyperkeratosis [7, 8], and palmar-plantar erythrodysesthias $[7,19]$. In clinical trials and subsequent dermatology-reported cohorts, this palmoplantar eruption was reported to occur in $6.3-12.5 \%$ of patients treated with vemurafenib, 7-30\% with dabrafenib, and 0-9 \% with dabrafenib/trametinib combination (Table 1). Very little has been reported regarding time to onset of HFSR/palmar-plantar hyperkeratosis. Huang et al. reported the development of calluses and tenderness on plantar skin within the first month of vemurafenib therapy $[12 \bullet \bullet$.

Clinically, BRAFi-induced palmar/plantar eruptions have been reported to range from isolated tender heels $[11 \bullet \cdot]$, to mild asymptomatic hyperkeratosis at areas of pressure, to an entity often described with sorafenib therapy termed "hand-

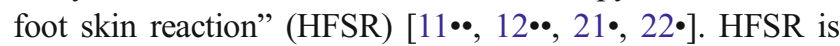
characterized by the development of thick, yellow, callus-like hyperkeratosis on a base of erythema at areas of pressure/friction on the soles, and less commonly on the palms $[9 \bullet, 12 \bullet \cdot, 22 \cdot]$. Although the term "palmar-plantar erythrodysesthesias" (PPE) has also been used in clinical trials to describe this BRAFi-induced entity [7], consensus among dermatologist authors $[9 \bullet, 21 \bullet]$ is that PPE is a different entity, reserved for the palmoplantar eruption occurring in the context of traditional chemotherapy agents, also referred to as "toxic erythema of chemotherapy" [38], characterized by diffuse palmoplantar edema, erythema, occasional blistering desquamation, and dysesthesias. In our experience, although erythema is noted in association with the hyperkeratosis in patients on BRAFi therapy, the overall reaction has been noted to be less inflammatory and diffuse than traditional toxic erythema of chemotherapy or multi-kinase inhibitor-induced HFSR. To date, BRAFi-induced HFSR/palmar-plantar hyperkeratosis has not been evaluated histologically.

Spontaneous improvement (of pain, hyperkeratosis, or both) has been noted by authors in the absence of treatment interruption or additions $[11 \bullet \bullet, 12 \bullet, 22 \bullet]$. Boyd et al. proposed a treatment algorithm that was extrapolated from sorafenib/sunitinib-induced HFSR literature [22•] (Table 2).

\section{Epidermal Cysts/Milia, Nodulocystic Acneiform Eruption}

In the last year, there have been an increasing number of reports of milia-like cysts, most commonly on the face, but also on the scalp and buttocks, occurring in the setting of selective BRAFi therapy $[9 \cdot \bullet, 11 \bullet, 12 \cdot \bullet$, $21 \bullet, 22 \bullet]$. Lesions are reported to start as small miliacyst like structures that can quickly progress to diffuse, large disfiguring nodulocystic lesions often with a head/neck distribution $[9 \cdot \bullet, 22 \bullet]$. Numerous grouped nodulocystic facial lesions have also been reported upon the bilateral face $[12 \bullet, 22 \bullet$, one in the setting of a KP-

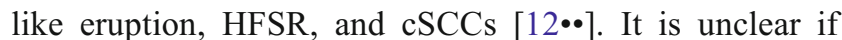
milia-like cysts and nodulocystic lesions reported are the same or different entities. There were no reports of histology for these eruptions. 
Milia-like epidermal inclusion cysts were not reported in clinical trials. Among subsequent dermatology-reported cohorts, milia-like cysts are reported to occur in $7-26 \%$ of patients treated with vemurafenib, $20 \%$ with dabrafenib, and $0 \%$ with dabrafenib/trametinib (Table 1), with a single case report citing time to onset of 4 months [12••].

In most reports, facial cystic lesions were not treated [21•], with reports of self-resolution [12••]. However, Boyd et al. reports a case of improved scalp lesions after 8 weeks of using a topical retinoid, and a case of improved facial lesions after 8 weeks of taking acitretin $10 \mathrm{mg}$ daily [22•] (Table 2).

\section{Hair Changes}

\section{Alopecia/Increased Hair Loss}

BRAFi-induced alopecia has been described as generalized thinning of scalp hair, with some reports of thinning of body hair [11••]. In clinical trials and subsequent dermatologyreported cohorts, alopecia was reported to occur in 36-70\% of patients treated with vemurafenib, 1-34 \% with dabrafenib, and 5-9\% with dabrafenib/trametinib combination (Table 1), with the mean time to onset of approximately 134 days in one report $[11 \bullet \bullet]$. No treatments were reported in the literature.

\section{Follicular Erythema and Hyperkeratosis}

Anforth et al. reported $9 \%$ (4/41) of patients developing follicular erythema and hyperkeratosis among a cohort of

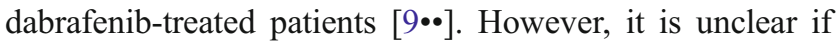
this was referring to scalp follicles, representing a lichenplanopilaris-like picture, or whether this observation was more consistent with a KP-like eruption as described above.

\section{Curly Hair}

Although not reported in clinical trials, patients with straight hair developing curly hair as a result of a selective BRAFi has been reported in $17 \%$ (7/41) of patients receiving dabrafenib [9••]. Similarly, in our clinical practice we have observed this finding (unpublished data), with hair relaxation occurring following unrelated discontinuation of therapy. It is unclear if this curling of the hair is reversible following discontinuation of therapy.

\section{Anti-CTLA4 Agents}

Introduction

Cytotoxic T lymphocyte-associated antigen 4 (CTLA4) is a protein that is expressed on the cell surface of activated $\mathrm{T}$ cells. The primary function of CTLA4 is to down-regulate T cell activation. Specifically, CTLA4 on activated T cells binds to co-stimulatory molecules (B7-1 and B7-2) on antigen presenting cells (APCs), blocking the production of IL2 , and leading to the arrest of $\mathrm{T}$ cell activation [2]. Regulatory T cells (Tregs) express CTLA4 at a much higher frequency $(50 \%$, compared with $<1 \%$ of naive CD4+ T cells), and their inhibition likely play a significant role in augmenting the $\mathrm{T}$ cell immune response in the setting of anti-CTLA4 therapy [2]. Under physiologic conditions, this regulatory mechanism is important for preventing excessive $\mathrm{T}$ cell mediated inflammation and autoimmunity; however, in the treatment of malignancy an enhanced and prolonged anti-tumoral T cell response is desirable [2]. Ipilimumab and tremelimumab are humanized monoclonal antibodies against CTLA4 that enhance T cell mediated anti-tumor response. Ipilimumab was the first drug of this class to show a survival benefit in the treatment of metastatic melanoma and was approved by the FDA in March 2011 [39, 40]. Although tremelimumab was found to have similar efficacy and side effect profile with ipilimumab, there is no plan for approval of tremelimumab to treat melanoma at this time [41].

The unique mechanism of action of anti-CTLA4 therapies differentiates them from standard chemotherapy or V600E-BRAF inhibitors, in that they target the immune system rather than the tumor itself, with the goal of overcoming immune tolerance in the setting of malignancy $[2$, $42 \bullet \cdot$. As a result, the main toxicities of anti-CTLA4 agents are autoimmune in nature. Although any organ can be affected, the most frequent immune-related side effects include: dermatitis (pruritus, rash, and vitiligo), colitis or hepatotoxicity, endocrinopathies, and neuropathies [43]. An increase in ANA and rheumatoid factor levels have also been noted, although the induction of generalized systemic autoimmunity does not typically occur [39, 44].

In the setting of ipilimumab monotherapy, immune-related cutaneous toxicity was reported to occur in 35-69\% of patients during clinical trials (Table 1), with an onset ranging from a few weeks to 10 months [44-46, 47••]. In clinical trials, these toxicities were sub-classified as rash, vitiligo, and pruritus [39], with subsequent reports of alopecia areata [42••] and hair depigmentation [47••]. Although anti-CTLA4 cutaneous toxicities are frequent, very little has been reported by dermatologists characterizing these eruptions.

\section{Rash}

In clinical trials and a subsequent dermatology-reported cohort, "rash" is reported to occur in 14-68\% of patients (Table 1 ) with $12 \%(65 / 520)$ being of moderate severity and $2.5 \%(13 / 520)$ considered life-threatening [40]. The time to onset for ipilimumab-induced rash varies in the literature from 1-4 weeks [44-46, 48] to a median time of 30 days 
(ranging 3-65 days) [42••]. Of note, nearly $50 \%$ of eruptions were noted following the first dose $[42 \bullet \bullet]$. The eruption has also been reported to occur very early in therapy or after therapy has ended, with the potential to worsen after each dose in some reports [49].

Clinically, the rash associated with anti-CTLA4 therapy has been described as a classic drug-induced morbilliform eruption. It is described as discrete, pruritic, pink-to-red, 2-4 mm erythematous papules that typically coalesce into thin plaques on the trunk and extremities [42••, 50•]. The eruption is more commonly located on proximal extensor limbs, followed by the trunk and distal extremities, with only occasional head and neck involvement, sparing the palms and soles [42••].

Histologically, anti-CTLA4 eruptions have also been likened to classic morbilliform drug eruptions in that they typically demonstrate a lymphocytic perivascular (and occasionally peri-adnexal) [42••, 50•]; dermal infiltrate, often accompanied by eosinophils $[41,42 \bullet \bullet, 50 \bullet$, with a CD4+ T cell predominance $[42 \bullet, 50 \bullet$. Mild spongiosis occurred in $67 \%(6 / 9)$, and mild $\mathrm{T}$ cell exocytosis in $56 \%(5 / 9)$, of samples $[42 \bullet \cdot]$.

Unique presentations of anti-CTLA4 induced rashes have also been reported, including the formation of lesions in areas of trauma (i.e. Koebner phenomena) [42••], one case of an urticarial morphology [49], one case of a symmetric photodistributed eruption [42・・], two cases of lightexacerbated eruptions [42••], a report of long-lasting injection site reactions in $17 \%$ (16/94) patients with 2-year survival [39], and one case of intertriginous plaques with concomitant follicular eruption of the trunk and groin [50 $]$. Hence, it has been suggested that anti-CTLA4 associated eruptions may appear clinically heterogeneous, but with a similar histologic pattern of hypersensitivity [50•]. However, at our institution, we have observed one biopsyconfirmed case of ipilimumab-induced neutrophilic dermatitis consistent with Sweet syndrome that presented 1.5 weeks after ipilimumab initiation, and a case of reactions at the site of a previous skin graft (personal communication from Junko Takeshita, M.D.). Furthermore, life-threatening eruptions have also been reported, with one death due to toxic epidermal necrolysis (TEN) [40].

The most common associations to occur with the antiCTLA4 eruption are pruritus (ranging from mild to severe $[42 \bullet \cdot]$ with occasional burning or pain $[42 \bullet \bullet, 51])$, and a peripheral eosinophilia (ranging from a $3-16$ fold increase) [42 ••, 50•]. No associated fevers or other systemic manifestations have been described. However, some authors have noted that cutaneous manifestations may predate other organ involvement [48]. Risk factors for developing anti-CTLA4 cutaneous dermatitis are unclear. There appears to be no association between a history of atopic dermatitis or psoriasis and the onset of eruption [42••]. However, potential risk factors may include: combination ipilimumab therapies (including dacarbazine [52], vaccines [39], or vemurafenib [31••]); male sex (7:2 male to female ratio) [42••]; or a history of cutaneous reactions to other medications (44\% in one cohort) [42••].

Median time to improvement to grade 1 or resolution has been reported to be 3.3 weeks [46], although it is unclear if this is in the setting of treatment or self-improvement. Few reports of successful treatments have been published, but include the following: topical steroids and topical antipruritics [49], treatment interruption along with either symptomatic treatment (emollients and diphenhydramine) [42••], or with a 30-day prednisone taper [51]. Monotherapy with diphenhydramine [53], a 2-week prednisone taper [50•], or complete discontinuation, have also been reported as successful. Only one treatment failure with the use of diphenhdramine and topical steroids has been reported [51]. Please refer to Table 2 for a consolidated empiric treatment algorithm from the literature.

\section{Pruritus}

In clinical trials and a subsequent dermatology-reported cohort, pruritus was the most frequent toxicity, occuring in 19$31 \%$ of patients treated with ipilimumab (Table 1), typically 3-6 weeks after treatment initiation [39, 43]. Anti-CTLA4induced pruritus is described to range from mild to severe in severity $[42 \cdot \bullet]$. No treatment modalities focused on treating pruritus are reported in the literature, but can be extrapolated from treatment reports for anti-CTLA4-induced eruptions.

\section{Vitiligo and Hair Depigmentation}

In clinical trials and subsequent cohorts, vitiligo and/or diffuse hair depigmentation are reported to occur in $2-14 \%$ of patients treated with ipilimumab or tremelimumab [39, 47••], with a median time to depigmentation of 10 months [47••]. Clinically, six cases of sudden hair depigmentation have been described. All cases started with eyebrow depigmentation that progressed to either complete body hair depigmentation or the development of diffuse vitiligo [47••]. In this same cohort, hair depigmentation or diffuse vitiligo was strongly associated with the development of other immune-related toxicities (e.g. rash, pruritus, diarrhea, or hypophysitis) and a durable response to therapy $[47 \bullet \bullet]$. In contrast to the development of diffuse vitiligo, localized vitiligo appearing preferentially around cutaneous metastases of melanoma has also been reported [54]. No specific treatment modalities have been reported in the literature.

\section{Alopecia Areata}

There were no reports of alopecia areata occurring in ipilimumab clinical trials. However, in a subsequent dermatologic cohort $1.6 \%(1 / 63)$ of patients treated with ipilimumab were reported to develop near-total non- 
scarring alopecia of the scalp, eyebrows, face, pubic region, and trunk, with a time to onset of approximately 50 days [42••]. Histologically, a diagnosis of alopecia areata was confirmed on scalp biopsy, demonstrating predominantly CD4+ peri-bulbar T cells and only scant CD $8+$ cells $[42 \bullet \cdot]$. Two months after treatment interruption, no improvement was noted $[42 \bullet \cdot]$. Otherwise, no other treatment modalities have been reported in the literature.

\section{Conclusion}

The treatment of metastatic melanoma has vastly changed over the last few years with the initiation of V600E-BRAF inhibitors and anti-CTLA4 agents. Given the widespread use these drugs in an outpatient setting, the role of outpatient dermatologists in monitoring and treating these eruptions will expand.

Conflict of Interest L Pappas-Taffer declares no conflicts of interest. M Rosenbach declares no conflicts of interest.

EY Chu declares no conflicts of interest.

\section{References}

Papers of particular interest, published recently, have been highlighted as:

- Of importance

•- Of major importance

1. Manousaridis I et al. Cutaneous side effects of inhibitors of the RAS/RAF/MEK/ERK signalling pathway and their management. J Eur Acad Dermatol Venereol. 2013;27(1):11-8.

2. Robert C, Ghiringhelli F. What is the role of cytotoxic T lymphocyte-associated antigen 4 blockade in patients with metastatic melanoma? Oncologist. 2009;14(8):848-61.

3. Davies $\mathrm{H}$ et al. Mutations of the BRAF gene in human cancer. Nature. 2002;417(6892):949-54.

4. Platz A et al. Human cutaneous melanoma; a review of NRAS and BRAF mutation frequencies in relation to histogenetic subclass and body site. Mol Oncol. 2008;1(4):395-405.

5. Chapman PB et al. Improved survival with vemurafenib in melanoma with BRAF V600E mutation. N Engl J Med. 2011;364 (26):2507-16.

6. Zelboraf ${ }^{\circledR}$ (vemurafenib) package insert. South Sn Francisco, CA: Genentech USA Inc.;2012.

7. Hauschild A et al. Dabrafenib in BRAF-mutated metastatic melanoma: a multicentre, open-label, phase 3 randomised controlled trial. Lancet. 2012;380(9839):358-65.

8. Flaherty KT et al. Combined BRAF and MEK inhibition in melanoma with BRAF V600 mutations. N Engl J Med. 2012;367 (18):1694-703.

9. •- Anforth RM et al. Cutaneous manifestations of dabrafenib (GSK2118436): a selective inhibitor of mutant BRAF in patients with metastatic melanoma. Br J Dermatol. 2012;167 (5):1153-60. Prospective dermatologic observational study of patients $(n=43)$ enrolled at a single institution in phase I/II dabrafenib trials in Sydney, Australia.
10. Alloo A et al. Photodynamic therapy for multiple eruptive keratoacanthomas associated with vemurafenib treatment for metastatic melanoma. Arch Dermatol. 2012;148(3):363-6.

11. .- Sinha $\mathrm{R}$ et al. Cutaneous adverse events associated with vemurafenib in patients with metastatic melanoma: practical advice on diagnosis, prevention and management of the main treatment-related skin toxicities. Br J Dermatol. 2012;167 (5):987-94. Review of cutaneous toxicities and dermatologic cohort of ten patients treated with vemurafenib.

12. •- Huang V et al. Cutaneous toxic effects associated with vemurafenib and inhibition of the BRAF pathway. Arch Dermatol. 2012;148(5):628-33. Cohort of 15 patients treated with vemurafenib.

13. • Chu EY et al. Diverse cutaneous side effects associated with BRAF inhibitor therapy: a clinicopathologic study. J Am Acad Dermatol. 2012;67(6):1265-72. Reported the cutaneous side effects of 14 patients treated with BRAFis.

14. $\mathrm{Su} \mathrm{F}$ et al. RAS mutations in cutaneous squamous-cell carcinomas in patients treated with BRAF inhibitors. N Engl J Med. 2012;366 (3):207-15.

15. Weeraratna AT. RAF around the edges - the paradox of BRAF inhibitors. N Engl J Med. 2012;366(3):271-3.

16. Oberholzer PA et al. RAS mutations are associated with the development of cutaneous squamous cell tumors in patients treated with RAF inhibitors. J Clin Oncol. 2012;30(3):31621.

17. Lacouture ME et al. Induction of cutaneous squamous cell carcinomas by RAF inhibitors: cause for concern? J Clin Oncol. 2012;30(3):329-30.

18. - Harvey NT, Millward M, Wood BA. Squamoproliferative Lesions Arising in the Setting of BRAF Inhibition. Am J Dermatopathol. 2012;34(8):822-6. Histologic review of squamoproliferative lesions in cohort of patients treated with BRAFis.

19. Sosman JA et al. Survival in BRAF V600-mutant advanced melanoma treated with vemurafenib. N Engl J Med. 2012;366(8):707-14.

20. Falchook GS et al. Dabrafenib in patients with melanoma, untreated brain metastases, and other solid tumours: a phase 1 dose-escalation trial. Lancet. 2012;379(9829):1893-901.

21. Mattei PL et al. Cutaneous effects of BRAF inhibitor therapy: a case series. Ann Oncol. 2012. Retrospective case review of 33 patients treated with BRAFis-15 patinets treated with vemurafenib, 18 patients treated with combination dabrafenib/ trametinib.

22. • Boyd KP et al. Nonmalignant cutaneous findings associated with vemurafenib use in patients with metastatic melanoma. J Am Acad Dermatol. 2012;67(6):1375-9. Review of non-malignant skin side effects associated with vemurafenib, providing pictures.

23. - Anforth $\mathrm{R}$ et al. Mutational analysis of cutaneous squamous cell carcinomas and verrucal keratosis in patients taking BRAF inhibitors. Pigment Cell Melanoma Res. 2012;25 (5):569-72. Evaluated frequency of RAS and PIK3CA in verrcous keratosis and cSCC.

24. Long GV et al. Dabrafenib in patients with Val600Glu or Val600Lys BRAF-mutant melanoma metastatic to the brain (BREAK-MB): a multicentre, open-label, phase 2 trial. Lancet Oncol. 2012;13(11):1087-95.

25. - Dalle S, Poulalhon N, Thomas L. Vemurafenib in melanoma with BRAF V600E mutation. N Engl J Med. 2011;365(15):1448-9. author reply 1450. First author in the literature to report new or evolving atypical nevi and new primary melanomas, found to have wild-type BRAF.

26. •- Zimmer L et al. Atypical melanocytic proliferations and new primary melanomas in patients with advanced melanoma undergoing selective BRAF inhibition. J Clin Oncol. 2012;30 (19):2375-83. Study evaluating genetic mutations and signal 
transduction molecule expression in 22 cutaneous melanocytic lesions that were clinically suspcious fro melanoma at seven international melanoma centers in clinical trials 2010-2011. Confirmed all were wild-type BRAF, with increased downstream MAPK pathway activation.

27. - Haenssle, H.A., et al., Dynamic Changes in Nevi of a Patient With Melanoma Treated With Vemurafenib: Importance of Sequential Dermoscopy. Arch Dermatol. 2012;148(10):1183-5. First to report three dynamic changes in nevi in a patient with sequential dermoscopy, and also showed the changing nevi or new nevi were wild type BRAF.

28. Dalle, S., et al. Second primary melanomas under vemurafenib. $\mathrm{Br}$ J Dermatol, 2012. doi:10.1111/bjd.12093.

29. Fearfield, L., et al. Second perimary melanomas under vemurafenib-reply from the author. Br J Dermatol, 2012. doi:10.1111/bjd.12094

30. • Dummer R, Rinderknecht J, Goldinger SM. Ultraviolet A and photosensitivity during vemurafenib therapy. N Engl J Med. 2012;366(5):480-1. Demonstrated vemurafenib-induced photosensitivity is UVA-mediated, and showed that it can be revered with UVA-blocking sunscreen.

31. • Harding JJ, Pulitzer M, Chapman PB. Vemurafenib sensitivity skin reaction after ipilimumab. N Engl J Med. 2012;366(9):866-8. Cohort of 12 paitents with sequential ipilimumab then vemurafenib had increased risk for morbilliform eruptions.

32. - Novoa RA et al. Vasculitis and panniculitis associated with vemurafenib. J Am Acad Dermatol. 2012;67(6):e271-2. Two cases of panniculitis without arthralgias, 1 case of vasculitis with ESS

33. - Gupta M et al. Unusual complication of vemurafenib treatment of metastatic melanoma: exacerbation of acantholytic dyskeratosis complicated by Kaposi varicelliform eruption. Arch Dermatol. 2012;148(8):966-8. Case of preexistant Grover's disease that worsened with vemurafenib, complicated by HSV superinfection.

34. - Story SG et al. Eccrine squamous syringometaplasia associated with vemurafenib therapy. J Am Acad Dermatol. 2012;67(5): e208-10. Reported facial papules with eccrine squamous syringometaplasia on biopsy.

35. Pappas-Taffer L, Higgins HW, Robinson-Bostom L, McDonald CJ. Dermatologic toxicities of anticancer therapy. In: Abeloff M, Armitage J, Niederhuber J, Kastan M, McKenna W. Gillies, editors. Abeloff's Clinical Oncology, 5th Edition. Philadelphia, PA: Elsevier; 2012.

36. - Zimmer L et al. Panniculitis with arthralgia in patients with melanoma treated with selective BRAF inhibitors and its management. Arch Dermatol. 2012;148(3):357-61. Reported two cases of panniculitis with arthralgias.

37. - Infante J, F.G., Lawrence D, et al.. Phase I/II study to assess safety, pharmacokinetics, and efficacy of the oral MEK $1 / 2$ inhibitor GSK1120212 (GSK 212) dosed in combination with the oral BRAF inhibitor GSK2118436 (GSK436) [abstract 8503]. J Clin Oncol, 2011;29 (suppl). Case of panniculitis in the setting of combination BRAF/MEK inhibitor.

38. Bolognia JL, Cooper DL, Glusac EJ. Toxic erythema of chemotherapy: a useful clinical term. J Am Acad Dermatol. 2008;59 (3):524-9.

39. Hodi FS et al. Improved survival with ipilimumab in patients with metastatic melanoma. N Engl J Med. 2010;363(8):711-23.

40. Yervoy $^{\mathrm{TM}}$ (ipilimumab) package insert. Princeton, NJ: BristolMyers Squibb Company; 2012.

41. Weber JS, Kahler KC, Hauschild A. Management of immunerelated adverse events and kinetics of response with ipilimumab. J Clin Oncol. 2012;30(21):2691-7.
42. •- Jaber SH et al. Skin reactions in a subset of patients with stage IV melanoma treated with anti-cytotoxic T-lymphocyte antigen 4 monoclonal antibody as a single agent. Arch Dermatol. 2006;142 (2):166-72. Morbilliform eruption noted prospectively in a cohort of 63 patients receiving monotherapy ipilimumab.

43. Zimmer $L$ et al. Side effects of systemic oncological therapies in dermatology. J Dtsch Dermatol Ges. 2012;10(7):475-86.

44. Weber $\mathrm{J}$ et al. A randomized, double-blind, placebo-controlled, phase II study comparing the tolerability and efficacy of ipilimumab administered with or without prophylactic budesonide in patients with unresectable stage III or IV melanoma. Clin Cancer Res. 2009;15(17):5591-8.

45. Wolchok JD et al. Ipilimumab monotherapy in patients with pretreated advanced melanoma: a randomised, double-blind, multicentre, phase 2, dose-ranging study. Lancet Oncol. 2010;11 (2):155-64.

46. O'Day SJ et al. Efficacy and safety of ipilimumab monotherapy in patients with pretreated advanced melanoma: a multicenter singlearm phase II study. Ann Oncol. 2010;21(8):1712-7.

47. • Pavlick AC OP, Kannan R, Madden KM, et al. Hair depigmentation as an indicator of durable response to CTLA-4 therapy. J Clin Oncol. 2010;28. doi:15s. (suppl; abstact 8571). Reported the occurance of hair depigmentation, often resulting in diffuse vitiligo, among a cohort of patients treated with anti-CTLA4 monoclonal antibody therapy.

48. Kahler KC, Hauschild A. Treatment and side effect management of CTLA-4 antibody therapy in metastatic melanoma. J Dtsch Dermatol Ges. 2011;9(4):277-86.

49. Gutzmer $\mathrm{R}$ et al. Cutaneous side effects of new antitumor drugs: clinical features and management. Dtsch Arztebl Int. 2012;109 (8):133-40.

50. • Lim JL, Dahiya M, Burgin S. Intertriginous and follicular eruption to anticytotoxic T-lymphocyte antigen 4 monoclonal antibody. J Am Acad Dermatol. 2008;59(2 Suppl 1):S60-1. Case report of eruption with ipilimumab and histologic findings.

51. Andrews S, Holden R. Characteristics and management of immunerelated adverse effects associated with ipilimumab, a new immunotherapy for metastatic melanoma. Cancer Manag Res. 2012;4:299-307.

52. Robert $\mathrm{C}$ et al. Ipilimumab plus dacarbazine for previously untreated metastatic melanoma. N Engl J Med. 2011;364(26):2517-26.

53. Di Giacomo AM, Biagioli M, Maio M. The emerging toxicity profiles of anti-CTLA-4 antibodies across clinical indications. Seminars in Oncology. 2010;37(5):499-507.

54. Sibaud V et al. Dermatologic adverse events of the new targeted anticancer therapies used in oncodermatology. Ann Chir Plast Esthet. 2012;57(2):106-13.

55. Flaherty KT et al. Inhibition of mutated, activated BRAF in metastatic melanoma. N Engl J Med. 2010;363(9):809-19.

56. Margolin K. Ipilimumab in a phase II trial of melanoma patients with brain metastases. Oncoimmunology. 2012;1(7):1197-9.

57. Beck KE et al. Enterocolitis in patients with cancer after antibody blockade of cytotoxic T-lymphocyte-associated antigen 4. J Clin Oncol. 2006;24(15):2283-9.

58. Viros, A., et al. Topical 5-Fluorouracil Elicits Regressions of BRAF Inhibitor-Induced Cutaneous Squamous Cell Carcinoma. J Invest Dermatol. 2012;133:274-76.

59. Anforth R et al. Systemic retinoid therapy for chemoprevention of nonmelanoma skin cancer in a patient treated with vemurafenib. J Clin Oncol. 2012;30(19):e165-7.

60. Fox MC et al. Management options for metastatic melanoma in the era of novel therapies: a primer for the practicing dermatologist: part II: management of stage IV disease. J Am Acad Dermatol. 2013;68(1):13 e1-13 e13. 